\title{
ELECTROSTATIC ACTUATORS WITH MECHANICAL BRAKES
}

\author{
John D. Grade, Kevin Y. Yasumura, and Hal Jerman \\ Iolon, Inc. \\ San Jose, CA 95131
}

\begin{abstract}
New DRIE actuator systems have been developed which use a high-force, linear actuator to wrap a flexible brake band around the rigid brake shoe of a large-deflection, rotary actuator. The brake can hold the rotary actuator at any arbitrary location within its full range of travel. A third actuator may be used to engage a pair of locking pins in order to keep the brake motor extended in the event of a power failure. The $100 \times$ increase in rotational stiffness after the brake is applied raises the fundamental resonant frequency by more than an order of magnitude, enabling precision optical adjustments over a large tuning range in the presence of external shock and vibration.
\end{abstract}

\section{INTRODUCTION}

With the improved stability from advances in suspension design, comb drive actuators are being deployed in applications requiring ever larger deflections. With total ranges in the hundreds of microns, the sub-micron positioning required for many applications is increasingly difficult to achieve, especially with the shocks and vibrations experienced in a product environment. This problem is commonly addressed in macroscopic applications by mounting small-deflection end effectors to large-deflection coarse positioners. However, this is less attractive with micromachined electrostatic actuators due to the additional fabrication complexity involved. An actuator with a mechanical brake provides similar functionality by increasing the suspension stiffness of the system to accomplish fine positioning once the coarse position has been set. Moreover, the brake may be used to implement a latching function, maintaining the actuated position of the motor in the event of a power failure or in applications requiring zero steady-state power.

As shown schematically in Fig. 1, DRIE actuators have been developed with mechanical braking mechanisms somewhat analogous to a drum brake in an automobile. Initial contact occurs between the vertical sidewalls in the center of the brake band, and the contact area increases as the brake actuator causes the band to wrap around the brake shoe.

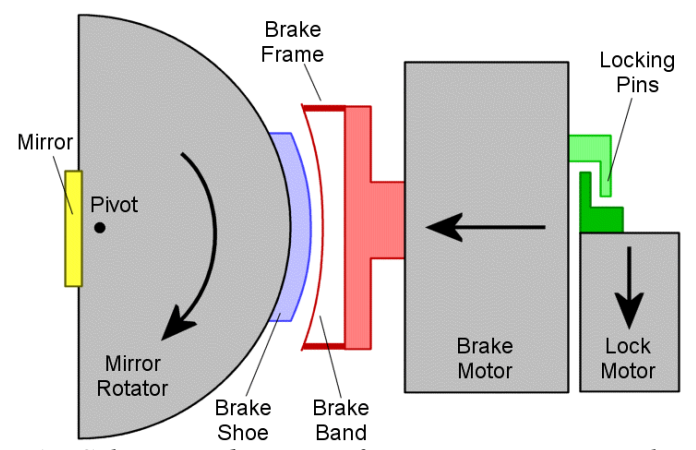

Figure 1. Schematic diagram of a rotary actuator with a brake mechanism. A pair of locking pins can hold the brake motor in place in the event of a power failure. Compliance in the brake frame can allow a small amount of mirror rotation after the brake is applied.
DRIE actuators have been developed with the mechanical braking mechanisms shown schematically in Fig. 1. In one example, the brake forms part of a latching optical switch, maintaining the actuated position of the motor in case of a power failure. Another example uses a brake to accomplish precision optical adjustments over a large tuning range in the presence of external shock and vibration. Unlike other power-safe schemes for electrostatic actuators [1-3], the mirror rotator in Fig. 2 may be held at any arbitrary location within its full range of travel.

\section{DESIGN}

The fabrication of the devices is identical to that used for optical devices described at $\mathrm{HH} 2002$ [4]. A cavity formed in an oxidized carrier wafer determines which portions of the final structure are fixed to the substrate and which are free to move. A second wafer is fusion bonded to the etched side of the carrier wafer and then ground and polished to a thickness of $85 \mu \mathrm{m}$. After contact holes and aluminum bonding pads are defined, the $85 \mu \mathrm{m}$ thick silicon layer is DRIE etched to form the suspended electrostatic actuator, substrate anchored areas, bond pads, and electrical interconnects. The devices typically feature $4 \mu \mathrm{m}$ wide suspension beams and $10 \mu \mathrm{m}$ wide comb gaps, which are reliably obtained using available etching tools.

Fig. 2 shows a set of actuators for a latching optical switch [5, 6] which includes a mirror rotator, a brake motor, and a locking motor. After the mirror rotator has set the desired mirror rotation, the lock motor is retracted and the brake motor is extended. The flexible brake band wraps around the rigid brake shoe and inhibits further mirror rotation. Once the brake has been applied, the lock motor may be released. A single bi-stable actuator could replace the brake and lock motors [7], but this would provide little control over the force transition as the brake is applied.

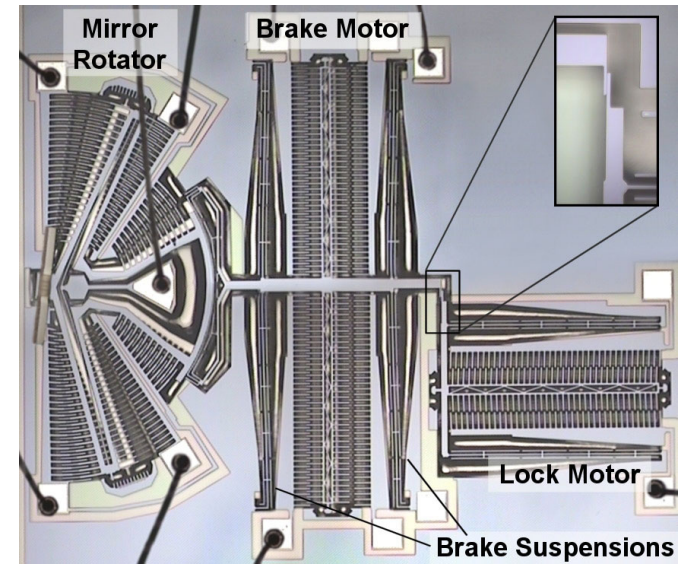

Figure 2. Microscope photograph of an actuator for a latching optical switch, similar to the diagram in Fig. 1. The locking motor is holding the brake motor in the deflected position, and the brake band and shoe are in contact with the mirror held at a rotation of 5 degrees. 
The motor device layer is $85 \mu \mathrm{m}$ thick and the mirror (mounted out-of-plane) is $600 \times 600 \times 85 \mu \mathrm{m}^{3}$. The mirror rotator has a range of $\pm 6^{\circ}$ with $130 \mathrm{~V}$ and a resonant frequency of $900 \mathrm{~Hz}$. The brake actuator requires $110 \mathrm{~V}$ to press the brake band into contact with the brake shoe.

The radius of the brake shoe was set to $80 \%$ of the brake band to ensure that the band wrapped around the shoe during operation. The contact area begins as a vertical line at the center of the brake band and grows with increasing brake motor force to include the entire band length.

Ideally, the activation speed of the brake motor would be much faster than that of the mirror rotator, the brake band would not move laterally when extending, and the motor would be essentially rigid once the brake has been applied. These requirements make a high-force, small-displacement actuator attractive; however, the fabrication process requires at least a 10 $\mu \mathrm{m}$ gap between the brake band and the brake shoe. The brake motor shown in Fig. 2 uses a symmetric, crab-leg suspension for lateral rigidity and linear actuation. Finite element analyses were used to determine suspension widths that brought the brake into first contact at $110 \mathrm{~V}$ and finished wrapping the band around the shoe after $150 \mathrm{~V}$ had been applied. To aid in overcoming potential stiction forces between the brake band and shoe, the initial brake motor designs featured opposed comb banks for bidirectional actuation, as shown in Fig. 2. As no stiction was observed, subsequent designs used single-sided actuation.

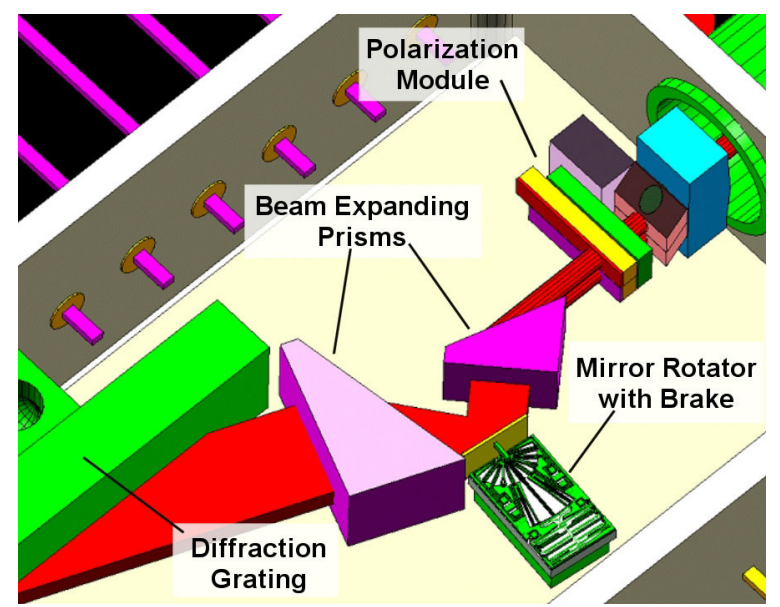

Figure 3. Schematic drawing of a tunable optical filter containing a rotary actuator with a mechanical brake. A laser beam enters and exits a fiber pigtail. Rotating the mirror changes the retroreflected angle of the beam on a grating, changing the center frequency of the filter.

Fig. 3 and Fig. 4 show a tunable optical filter containing a rotary actuator with a mechanical brake that is used for coarse and fine position control rather than for power-safe operation [8]. The coarse servo system sets the initial rotation of the mirror, and then the brake is applied. The compliance of the frame supporting the brake band was designed to allow the mirror to be adjusted by $1 \%$ of the full range after braking, enabling precision optical adjustments in the presence of external shock and vibration while maintaining the large tuning range.

The optical design for the tunable filter requires an out-ofplane mirror that is quite large $\left(2500 \times 1270 \times 85 \mu^{3}\right)$. Since the motor device layer is only $85 \mu \mathrm{m}$ thick, two different tactics are employed to reduce the out-of-plane vibration response. First, as shown in Fig. 5, the mirror is mounted off the left side of the chip, and it extends above and below the motor along the z-axis. This reduces the mirror's out-of-plane inertia by a factor of 4 and causes the mirror and motor masses of the resulting system to balance each other about all three axes. The downside is a relatively complex two-piece attachment between the mirror and the motor. Second, the mirror rotator includes flexural suspension beams at the pivot point ( \pm 70 degrees from the long axis of the chip) and at the center of the device ( \pm 15 degrees from the long axis). Partially compliant portions of the outer frame accommodate differences in suspension foreshortening during motor rotation. This arrangement provides excellent stiffness in both pitch and roll. It also allows the brake to be applied far from the pivot point, reducing the tendency of the brake to slip due to any torques induced by external shock or vibration.

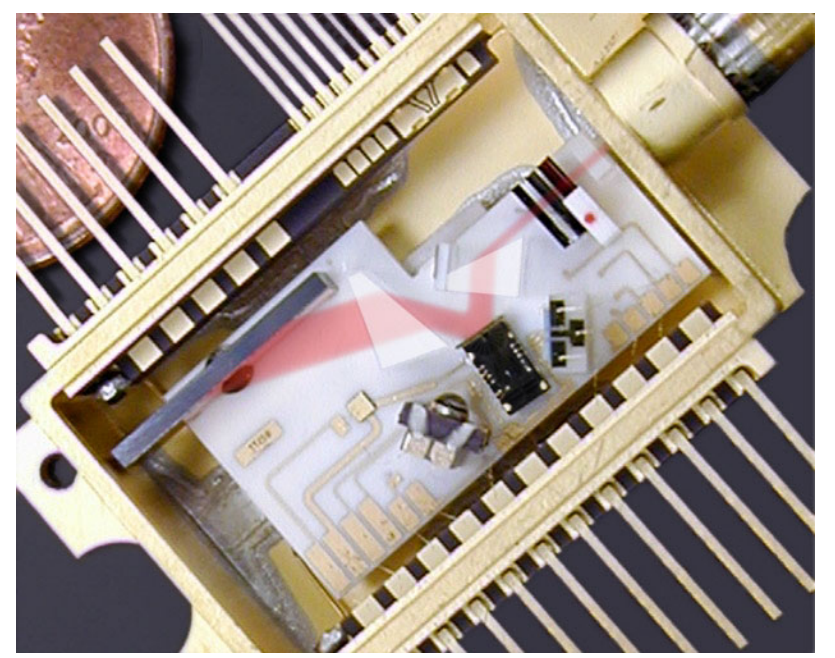

Figure 4. Assembled tunable optical filter containing a rotary actuator with a mechanical brake (center).

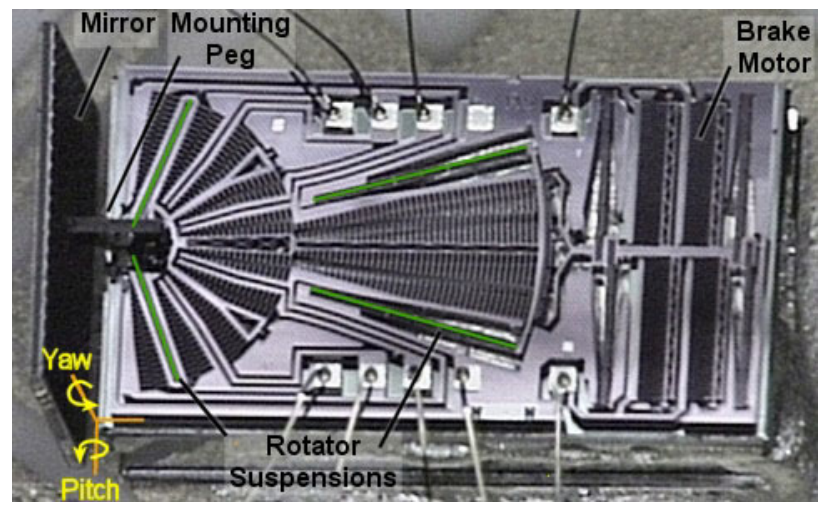

Figure 5. An example actuator for a tunable filter in which the brake mechanism is used for fine positioning and vibration rejection.

\section{EXPERIMENTAL RESULTS}

The performance of the rotary actuator in Fig. 2 was verified using a $670 \mathrm{~nm}$ laser reflected off the out-of-plane mirror onto a position sensitive detector (PSD). Voltage was first applied to the mirror rotator to set the desired mirror angle as shown in Fig. 6. A cosine-shaped voltage transition was used in order to reduce ringing in the mirror position. Voltage was then applied to the brake motor to press the brake band firmly against the brake shoe. Finally, the voltage was removed from the mirror rotator, with the mirror angle maintained only by the brake. 
A small amount of compliance in the brake frame allowed a shift in mirror angle of $0.2 \%$ of the full-scale deflection when the mirror rotator was deactivated. As shown in the photograph in Fig. 2, the locking motor could also be used to keep the brake engaged when all voltages were removed.

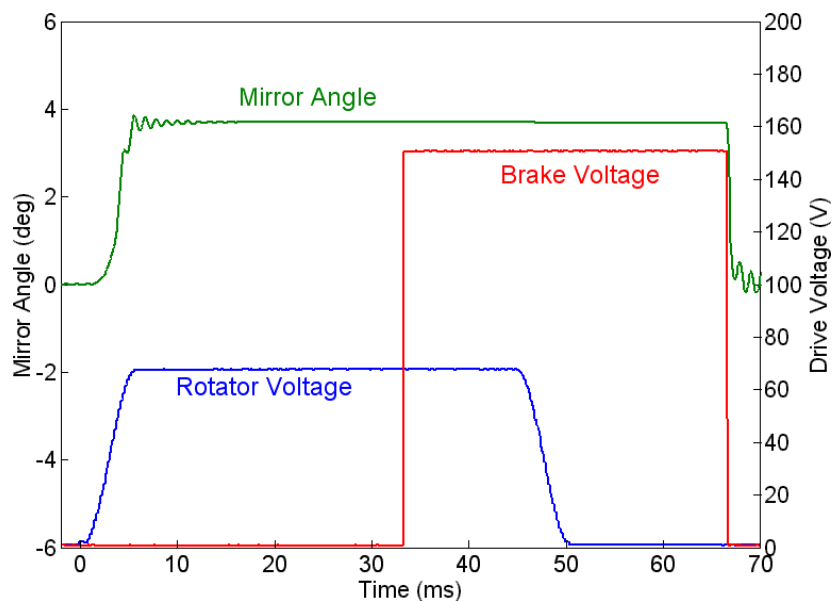

Figure 6. Example operation of the brake mechanism in Fig 2. After the mirror angle was set, the brake was applied, and the voltage was removed from the mirror rotator.

The same PSD measurement setup was used to characterize the actuator for the tunable optical filter. For this application, the brake was intended to improve resistance to shock and vibration by reducing the low frequency response by at least $100 \times$, and by raising the fundamental resonance by an order of magnitude.

Fig. 7 shows the change in mirror rotator frequency response with braking. The brake reduced the DC rotational stiffness by $160 \times$ and raises the fundamental resonance above from $850 \mathrm{~Hz}$ to $12.5 \mathrm{kHz}$. The out-of-plane resonant frequency rose by almost a factor of two, from $3.3 \mathrm{kHz}$ to $5.8 \mathrm{kHz}$.

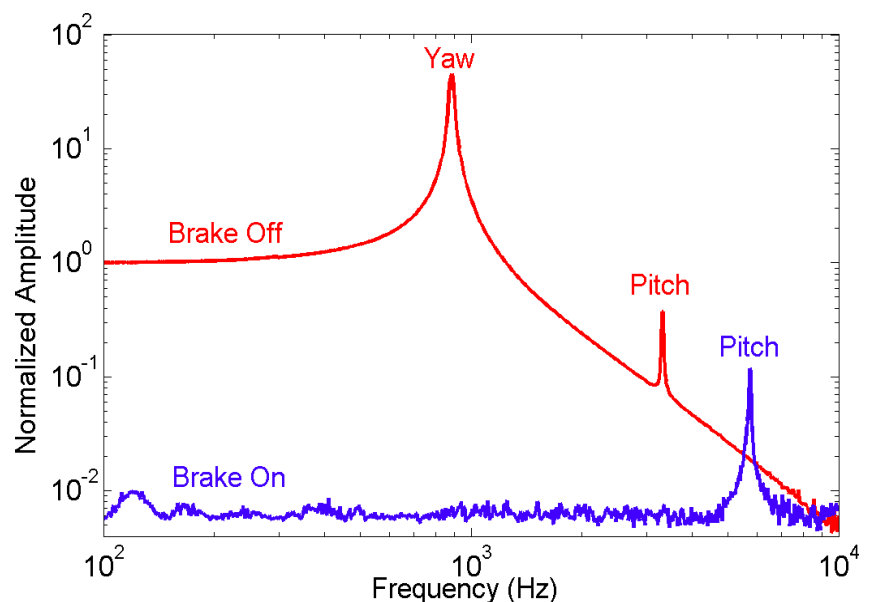

Figure 7. Measured, open-loop, frequency response of a tunable filter actuator. Applying the brake raised the fundamental resonant frequency (mirror yaw) from $850 \mathrm{~Hz}$ to $12.5 \mathrm{kHz}$ and raises the second mode (mirror pitch) from $3.3 \mathrm{kHz}$ to $5.8 \mathrm{kHz}$.

Several factors may combine to introduce errors in the final mirror position after the brake has been applied. Large radial loads applied by the brake may induce motion in the mirror rotator due to buckling of the individual suspension beams or compliance of the mirror motor frame. These errors should be a continuous function of the angle of the mirror motor, and they should be symmetric about the rest position. Discretization of the curves defining the brake may create facets that snap together and change the mirror angle slightly. Similarly, sidewall roughness arising from the DRIE step may cause the brake to engage preferentially at locations where the brake shoe and band have matching asperities. These sidewall effects would create angular shifts that are not correlated to the mirror position. The fine servo controller could compensate for angular shifts below $1 \%$ of the full-scale deflection, or $38 \mathrm{mdeg}$.

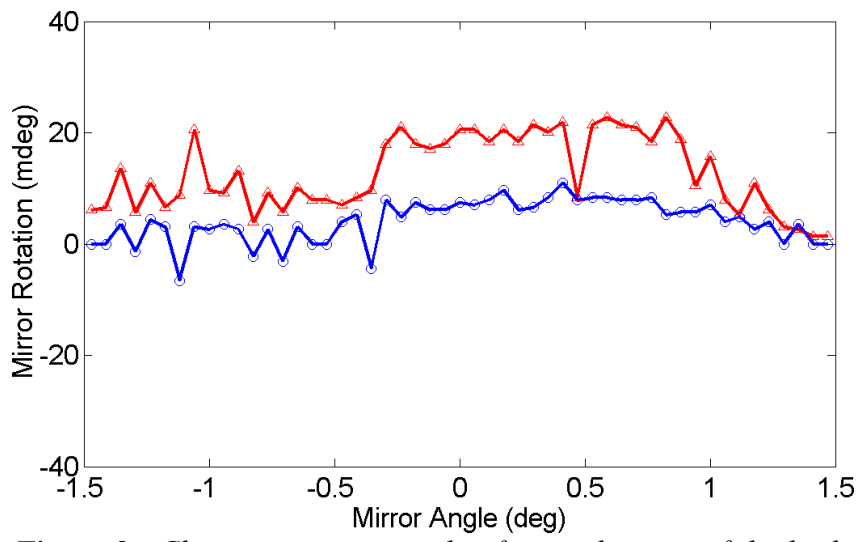

Figure 8. Change in mirror angle after application of the brake as a function of initial mirror position. The brake was driven sinusoidally with amplitudes of $150 \mathrm{~V}$ (triangles, top curve) and $90 \mathrm{~V}$ (circles, bottom curve). Brake slip occurred at $75 \mathrm{~V}$.

Fig. 8 shows a measurement of the post-brake angular shift as a function of mirror angle. The brake actuator was driven with a sinusoidal waveform to reduce the chances of the brake microbouncing when engaged at high speed. Measurements using a square wave showed no angular dependence and more than $5 \times$ the average angular shift. For both waveforms, lowering the brake force reduced post-brake angular shift, with the $90 \mathrm{~V}$ sinusoid yielding a maximum angular shift of $10 \mathrm{mdeg}$.

To more closely examine the effect of the brake force on the angular shift, the brake was applied repeatedly using a voltage that increased from $80 \mathrm{~V}$ to $150 \mathrm{~V}$. As shown in Fig. 9, the post-brake shift increases with increasing brake voltage.

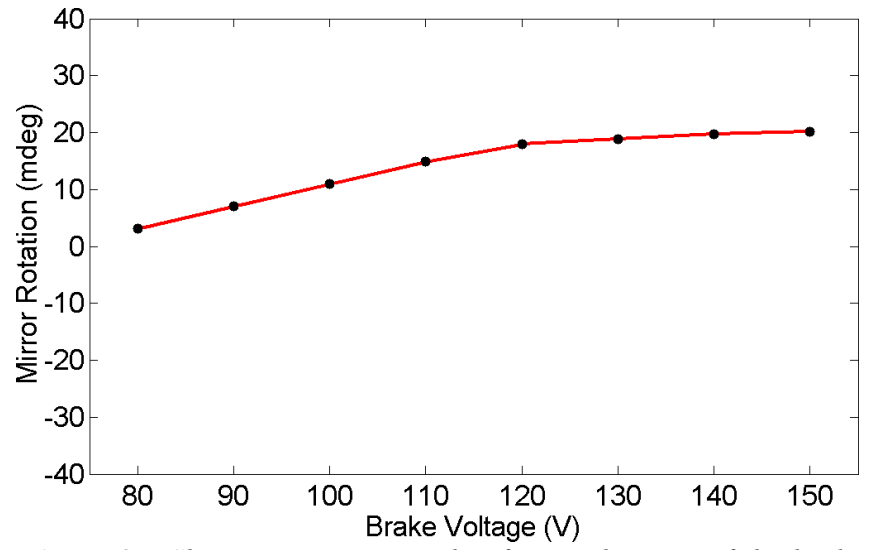

Figure 9. Change in mirror angle after application of the brake as a function of the brake voltage. The initial mirror angle was set to the center position, or zero degrees.

Two potential failure mechanisms, brake slip and brake stiction, have also been investigated. Brake slip is directly related to the coefficient of static friction between the brake shoe and 
brake band, which likely depends on the DRIE sidewall roughness. Fig. 10 shows the calculated electrostatic forces for the mirror rotator and brake actuator at the brake slipping point. Mirror rotator forces of approximately $60 \mu \mathrm{N}$ (torques of $0.17 \mathrm{~mm}-\mathrm{mN}$ ) caused slipping with the brake activated and caused full-scale deflection $\left( \pm 6^{\circ}\right)$ with the brake deactivated. Only shocks exceeding 5000 g's would generate forces sufficient to cause brake slip in mechanically balanced rotators [4]. A linear fit to the data yields a friction coefficient of 0.67 , which is within the range of reported values [9].

In the course of generating the friction measurements, it became clear that the friction coefficient could be raised by repeatedly overdriving the main actuator with the brake applied. This had the effect of removing small asperities, essentially burnishing the brake sidewall surfaces. The coefficient of friction rose quickly for the first few kilo-cycles and stopped rising after a few hundred kilo-cycles. Measurements of the slip voltage before and after burnishing indicated that the coefficient of friction had risen to 1.5 and that less than 0.5 microns of material had been removed.

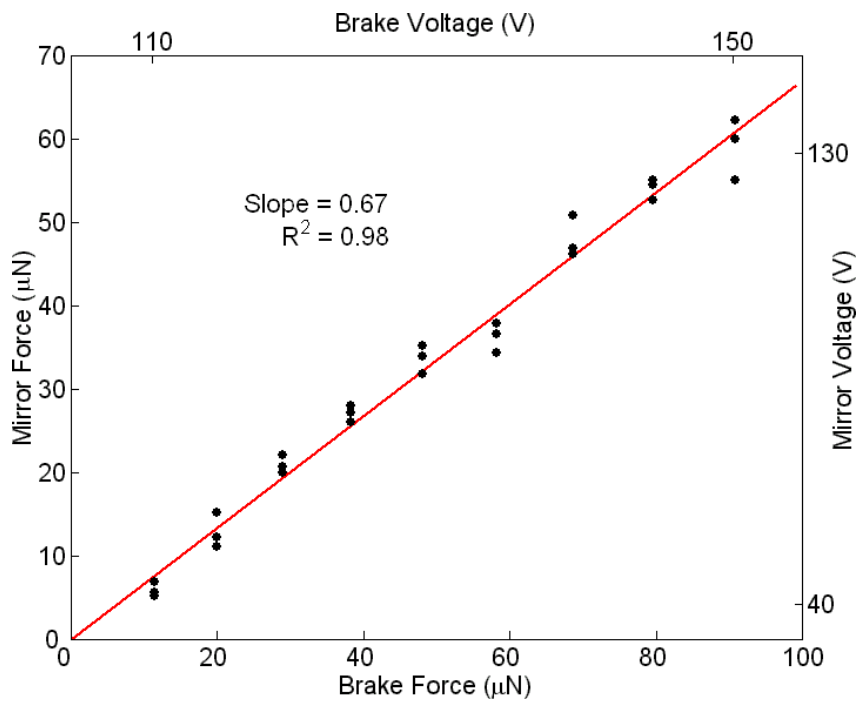

Figure 10. Relationship between the mirror rotator force and the brake motor force at which a burnished brake begins slipping. The slope of the least squares fit through the data indicates a coefficient of static friction of 0.67 .

Stiction is another concern when using a mechanical brake. To reduce stiction forces, the maximum contact area between the brake band and shoe was kept small (less than $0.04 \mathrm{~mm}^{2}$ ), and the brake band was designed to wrap on and peel off of the brake shoe. No hysteresis was observed in the quasi-static transfer function for the brake actuator, suggesting stiction-free operation. To simulate a high-humidity environment and guarantee that a liquid meniscus formed between the brake band and shoe, a very small amount of water was manually applied to the brake. The suspensions of the brake actuator readily overcame the capillary forces during this worst-case test.

\section{CONCLUSIONS}

A new strategy has been demonstrated for achieving precision optical adjustments in the presence of external shock and vibration without sacrificing total deflection range. The use of mechanical brakes allows an actuator to be held at any arbitrary location and can raise the fundamental resonant frequency by more than an order of magnitude. Burnishing and using a low brake force can reduce brake-induced motor rotation to levels that can be compensated by servo control.

\section{ACKNOWLEDGEMENTS}

The authors would like to thank C. Tan and K. Jackson on the iolon development team for their help in preparing and testing the actuator devices.

\section{REFERENCES}

1. A. Neukermans and R. Ramaswami, "MEMS Technology for Optical Networking Applications," IEEE Communications Magazine, 39, January 2001, pp. 62-69.

2. R. Yeh, S. Hollar, and K. Pister, "Single mask, large force, and large displacement electrostatic linear inchworm motors," Proceedings of the $14^{\text {th }}$ Annual International Conference on Microelectromechanical Systems, Interlaken, Switzerland, January 2001, pp. 260-264.

3. M. S. Rodgers and J. J. Sniegowski, "5-level polysilicon surface micromachine technology: application to complex mechanical systems," Technical Digest 1998 Solid State Sensor and Actuator Workshop, Hilton Head Isl., SC, June 1998, Transducer Research Foundation, Cleveland (1998), pp. 144-149.

4. H. Jerman and J. D. Grade, "A mechanically-balanced, DRIE rotary actuator for a high-power tunable laser," Technical Digest 2002 Solid State Sensor and Actuator Workshop, Hilton Head Isl., SC, June 2002, Transducer Research Foundation, Cleveland (2002), pp. 7-10.

5. J. D. Grade, H. Jerman, and T. W. Kenny, "A large-deflection electrostatic actuator for optical switching applications," Technical Digest 2000 Solid State Sensor and Actuator Workshop, Hilton Head Isl., SC, June 2000, Transducer Research Foundation, Cleveland (1994), pp.97-100.

6. H. Jerman, US patent publication number 20030034709, "Micromechanical device having braking mechanism," February 2003.

7. B. Hichwa et al., "A Unique Latching 2x2 Fiber Optics Switch," Proc. 2000 IEEE/LEOS Int'l. Conf. Opt. MEMS, Kauai, HI, August 2000, post-deadline paper.

8. J.D. Berger, F. Ilkov, D. King, A. Tselikov, and D. Anthon, "Widely tunable, narrow optical bandpass Gaussian filter using a silicon microactuator," in Optical Fiber Communication Conference, OSA Technical Digest, (OSA, Washington, DC, 2003), TuN2.

9. K. Deng and W. H. Ko, "A study of static friction between silicon and silicon compounds," J. Micromech. Microeng. 2, March 1992, pp. 14-20. 